\title{
Paediatric Crossword Puzzle 34
}

\section{Manouri P Senanayake ${ }^{1,2}$, A S Athapathu ${ }^{2}$}

Sri Lanka Journal of Child Health, 2017; 46(1): 95

DOI: http://dx.doi.org/10.4038/sljch.v46i1.8231

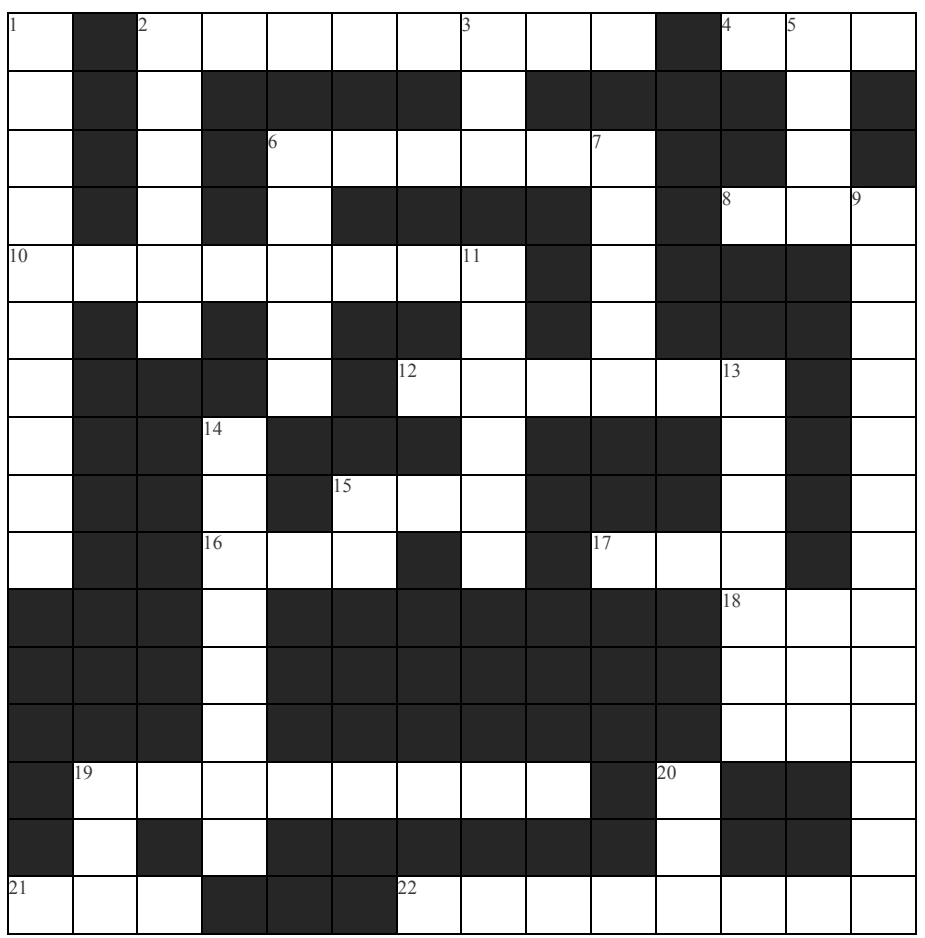

\section{$\underline{\text { ACROSS }}$}

2. Seizure characterized with laughter or crying

4. Essential steps in basic life support (abbrv.)

6. Route of midazolam administration to stop seizure

8. Skin and mucous membranes suggesting Kawasaki disease

10. Postnatal depression that is often missed 12. Milk intake associated with iron deficiency 15. A dangerous food allergy associated with delayed dietary exposure

16. Biochemical investigation used to screen for coeliac disease (abbrv.)

17. A cause of respiratory distress in the newborn (abbrv.)

18. A muscle disorder transmitted on the $X$ chromosome (abbrv.)

19. Gene mutation most commonly associated with inherited deafness

21. Increases risk of multiple pregnancies

22. Treats excessive salivation

\section{DOWN}

1. Faecal incontinence

2. Neuro-metabolic abnormalities are associated with reduced ..............

3. Tourette syndrome is a disorder involving discrete muscle groups

5. Acute lymphoblastic leukaemia may present with . pain

6. Viruses are spread through migration

7. Anterior fontanelle that warrants assessment for maternal Vitamin D deficiency

9. Anti-emetic linked with cardiac side effects

11. Joint abnormality seen in autism

13. The most commonly present sensory hypersensitivity in Autism Spectrum Disorder

14. Vitamin D deficiency can flare up tuberculosis

15. An effective route for correcting dehydration due to diarrhoea

19. Intrauterine infection that can cause deafness

20. Pneumoperitoneum in preterm babies (abbrv)

${ }^{1}$ Department of Paediatrics, Faculty of Medicine, Colombo, ${ }^{2}$ Lady Ridgeway Hospital for Children, Colombo 